\title{
René Maran - Jean-Joseph Rabearivelo
}

La même froide colère

\section{Claire Riffard}

\section{CpenEdition}

\section{Journals}

Édition électronique

URL : https://journals.openedition.org/coma/7109

DOI : 10.4000/coma.7109

ISSN : 2275-1742

Éditeur

Institut des textes \& manuscrits modernes (ITEM)

\section{Référence électronique}

Claire Riffard, «René Maran - Jean-Joseph Rabearivelo », Continents manuscrits [En ligne], 17 | 2021 , mis en ligne le 15 octobre 2021, consulté le 12 janvier 2023. URL : http://journals.openedition.org/ coma/7109; DOI : https://doi.org/10.4000/coma.7109

Ce document a été généré automatiquement le 12 janvier 2023.

\section{(c)}

Creative Commons - Attribution - Pas d'Utilisation Commerciale - Pas de Modification 4.0 International - CC BY-NC-ND 4.0

https://creativecommons.org/licenses/by-nc-nd/4.0/ 


\section{René Maran - Jean-Joseph Rabearivelo}

La même froide colère

Claire Riffard

\section{NOTE DE L'ÉDITEUR}

Cet article propose quelques pages du livre à paraître en 2022, Jean-Joseph Rabearivelo. Une biographie, chez CNRS Éditions.

1 Le 22 juin 1937, juste avant de se donner la mort dans son île australe, à 34 ans seulement, l'écrivain malgache Jean-Joseph Rabearivelo écrivait à René Maran :

Cher et lointain ami,

Cette lettre est la dernière que vous recevrez de moi.

Dans quelques heures, je ne serai plus qu'un cadavre.

Et la terre tournera toujours.

Et il y aura toujours des poètes pour chanter, indifférents à cette chose monstrueuse : LA MATIÈRE.

Votre

Jean-Joseph RABEARIVELO.

2 Maran répondait, avec émotion, dans un hommage posthume publié dans Le Monde illustré $^{1}$, qui rend bien compte de ses positions du temps en matière politique et littéraire.

\section{Rabearivelo selon Maran : « Un petit-neveu de Chatterton »}

Contrairement à ce que l'on croit, la plus grande France ne se limite pas qu'aux territoires que la France fait valoir sous tous les cieux. Elle englobe aussi nombre de collectivités étrangères. Celles-ci, estimant que la civilisation française est la plus belle qui soit, ne demanderaient pas mieux que de devenir les démarcheuses 
intellectuelles de la France. Encore faudrait-il qu'elle y consentît. Il serait de son intérêt de le faire. Ce n'est pas en vain qu'on parle français en Suisse, en Égypte, en Syrie, à l'île Maurice, au Canada et en Haïti.

Il suffirait d'un léger effort d'attention et de compréhension pour adjoindre à ces collectivités, les collectivités noires qui prolifèrent aux États-Unis et comptent présentement près de quatorze millions d'âmes. Évoluant en vase clos, ou plutôt en race close, elles voudraient que la France les aidât à s'évader du racisme où on les oblige à se murer. Il ne semble pas qu'on ait jusqu'ici entendu leur appel. Mais comment aurait-on pu l'entendre, puisque les lettres françaises ont fait, pendant seize ans, la sourde oreille aux chants que le poète malgache Jean-Joseph Rabearivelo se plaisait à consacrer à la défense de la langue française et à son illustration?

Quelques lettrés suivaient de loin le tenace, patient, solitaire et méritoire effort de Jean-Joseph Rabearivelo. Mme la baronne de Brimont, Jean Vignaud, Pierre Camo, Gaston Rageot, Maurice Martin du Gard, Jean Valmy-Baysse - et d'autres encore sans doute - étaient du nombre. La Vie, que les frères Marius-Ary Leblond dirigent avec tant de lucide et fervente allégresse, Le Divan, Les Nouvelles Littéraires et La Dépêche de Toulouse avaient publié ses poèmes ou des articles concernant ses ouvrages. Il n'était donc pas un inconnu. Il avait « la foi, la flamme, la religion par laquelle une âme devient légion ». On admirait non seulement son courage, mais encore l'ardeur et la conviction qu'il apportait à défendre tout ce qui était français, en dépit des sarcasmes dont le couvraient parfois certains de ses compatriotes.

On lui savait gré de son attitude. Il savait qu'on lui en savait gré. Il savait aussi qu'il comptait, en France, des amitiés agissantes. Il savait enfin qu'il avait du talent. Mais peut-être, à cause de cela, a-t-il rêvé d'un trop haut destin ?

Ce trop haut destin n'était cependant pas irréalisable. Jean-Joseph Rabearivelo ne ressemblait pas encore à ce vieux sculpteur de sons de La Sandale Ailée, à qui Henri de Régnier a fait dire qu'il

N'avait jamais désiré d'autre gloire

Sinon que les vers du poète

Plussent à la voix qui les répète.

Le renoncement est en général un privilège de l'âge. Et toute sagesse se fonde plus ou moins sur le renoncement. L'auteur de Chants pour Abéone n'en était pas là. Ignorant la vanité de la plupart des succès littéraires, il briguait, depuis des années, tantôt tel prix de poésie, tantôt tel autre.

Il avait failli avoir le prix Edgar Poe en 1934. Son échec lui avait été sensible. Il ne s'en était consolé qu'en essayant d'en avoir un autre, qui lui eût permis, selon l'expression de Léon Deubel, de « pousser ses vers sur le monde futur ».

Un second échec, qu'il a probablement attribué au mépris ou à l'indifférence, paraît l'avoir touché plus que de raison. Des chagrins familiaux, des soucis matériels, des deuils et la maladie sont venus accroître sa lassitude romantique. C'était plus que n'en pouvait supporter ce lointain petit-neveu de Chatterton. Il s'est tué parce qu'il aimait trop la poésie et qu'elle lui refusait les faveurs qu'il lui avait demandées.

Poète, il l'est resté jusqu'à ses derniers moments. Romantique, il a su quitter la vie en beauté. [...]

Comment ne pas se sentir les yeux pleins de larmes devant la gentillesse anthume de ce poète français, qui n'a pas voulu aller rejoindre ses ancêtres au pays des Mânes, sans prendre auparavant congé des écrivains de France dont l'attentive amitié ou l'aide spirituelle avaient été si longtemps pour lui une raison de vivre?

Jean-Joseph Rabearivelo n'avait pas encore réussi, avant de mourir, à se défaire de l'influence des poètes qu'il avait pris pour modèles. Ce manque de personnalité, compte tenu de ses afféteries inutiles, ou, si l'on préfère, de son gongorisme, était le seul reproche qu'on fût en droit de lui adresser.

Mais il commençait depuis peu à «se changer en lui-même». Il avait pris conscience de ses jeunes forces, en demandant à son pays natal d'inspirer son génie. Il allait bientôt voler de ses propres ailes. Les poèmes de Presque-Songes et ceux de 
Traduit de la Nuit en fournissent le témoignage - Presque-Songes, dont la page de garde de l'exemplaire que je possède porte cette dédicace :

$\grave{A}$

René Maran

ces recherches de la Poésie

faites loin des frontières de la langue française,

puis ramenées à Elle ;

et Traduit de la Nuit où, pour tout commentaire, j'ai noté, en juin ou juillet 1935, sur la page de garde du recueil, après avoir lu d'un bout à l'autre les poèmes qui le composent, cette exclamation de Pierre de Dace, 0 felix nox, o dulcis et delectabilis nox in qua mihi primum est degustare datum quam sit suavis Dominus !2

Triste conclusion d'une amitié exclusivement épistolaire mais néanmoins sincère et profonde, qui remonte aux années 1920.

\section{Maran selon Rabearivelo : « le grand Guyanais »}

Rabearivelo a-t-il eu connaissance à l'époque des articles de Maran publiés en 1921 dans le journal L'Action coloniale ${ }^{3}$ ? Peut-être. Mais un fait est certain : il a lu et relu Batouala, véritable roman nègre. Un choc. Redoublé en 1924 par la lecture des premiers numéros de la revue Les Continents, dirigée par Prince Kojo Tovalou avec la collaboration étroite de Maran, et surtout du conte Le Petit Roi de Chimérie. Découvrir de tels textes, trente ans à peine après la conquête française, alors que les blessures portées au fer rouge sont encore ouvertes, se sont infectées même, c'est pour un littérateur aussi impliqué que Rabearivelo dans sa société et son temps, un encouragement inespéré !

Il précise les raisons de son admiration dans sa réponse à une enquête sur les prix Goncourt ${ }^{4}$ menée en 1925 par la revue La Pensée latine ${ }^{5}$, choisissant d'afficher son soutien à Maran dans un contexte où ce dernier continuait d'être violemment mis en cause pour la préface de son roman. 


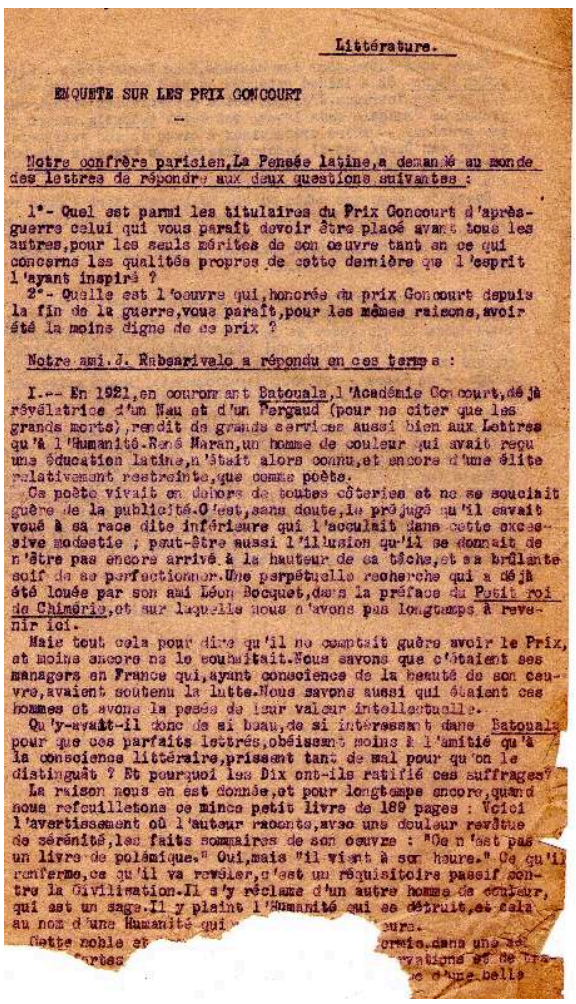

Archives Rabearivelo, NUM ETU TAP Goncourt.

Droits réservés

[...] Qu'y avait-il donc de si beau, de si intéressant dans Batouala pour que ces parfaits lettrés, obéissant moins à l'amitié qu'à la conscience littéraire, prissent tant de mal pour qu'on le distinguât ? Et pourquoi les Dix ont-ils ratifié ces suffrages? La raison nous en est donnée, et pour longtemps encore, quand nous refeuilletons ce mince petit livre de 189 pages : Voici l'avertissement où l'auteur raconte, avec une douleur revêtue de sérénité, les faits sommaires de son œuvre: "Ce n'est pas un livre de polémique ». Oui, mais « il vient à son heure ». Ce qu'il renferme, ce qu'il va révéler, c'est un réquisitoire passif contre la Civilisation. Il s'y réclame d'un autre homme de couleur, qui est un sage. Il y plaint l'Humanité qui se détruit, et cela au nom d'une Humanité qui [...].

Comme sous-titre, pour les badauds, René Maran écrit : Véritable roman nègre. On a laissé entendre qu'il l'est plus par son style que par son intrigue. J'estime cette appréciation éminemment saugrenue. Le français dans lequel est écrit Batouala, encore qu'un peu précieux - voire prétentieux à cause de son rutilant archaïsme (et, mon Dieu, est-il encore beaucoup de Français nés pour manier ces expressions tombées en désuétude aussi savamment que l'a fait Maran ?!) - est excellent.

Quand je songe que cette pureté de langage sert à nous révéler une sensibilité nouvelle et restitue à l'Humanité une part d'elle-même méconnue, quand je sais surtout que tant d'efforts réalisés ont été couronnés par les Goncourt, je crois avoir répondu à la première question ${ }^{6}$.

6 Au moment de se lancer dans l'arène romanesque, Rabearivelo conçoit les pages liminaires de son roman historique L'Aube rouge (1924-1925) exactement dans le même ton que la préface de Batouala. Avec la même froide colère.

7 Si les archives du poète ne conservent pas la trace du labeur d'incorporation au stade de l'avant-texte, la structure narrative du texte publié doit beaucoup à l'esthétique de Batouala. «Rabearivelo pose d'ailleurs, littéralement, son chevalet devant cette œuvre 
dont il s'inspire »; belle formule de Serge Meitinger, qui précise : "D'où, à l'imitation très fidèle des transitions utilisées par Maran dans son roman, les tableaux de la nature décrite surtout au lever et au coucher du soleil, les allusions appuyées au cycle lunaire et aux nuances climatiques, la description suggestive de la faune et de la flore, quelques paysages typiquement malgaches, visant souvent à symboliser comme celui des marais entourant le fort de Farafaty (qui signifie en malgache: "la mort ultime"), lieu de défaite et de mort pour les Français ${ }^{7}$. »

Rabearivelo dédicace à Maran ${ }^{8}$ ce roman qu'il ne parviendra jamais à publier. Il le met également à l'honneur dans la note liminaire (datée du 25 juillet 1925), où son nom avoisine ceux de Marcus Garvey, du Mahatma Gandhi (que Maran a contribué à faire connaître ${ }^{9}$ ) ou de Jean Ralaimongo.

\section{Une amitié au long cours}

9 Les deux hommes entament à cette époque une correspondance amicale qui ne s'éteindra qu'avec la disparition de Rabearivelo. Bien que les archives de l'un et de l'autre n'en conservent nulle trace, quelques fragments en sont heureusement rescapés dans Les Calepins bleus (CB), le journal personnel de Rabearivelo couvrant la période 1933 à 1937 de sa "vie écrite $»^{10}$. En mars 1935, l'écrivain malgache y accuse réception d'un courrier de son ami guyanais. Le 15 juin 1935 derechef, « une lettre de Maran [lui] annonçant le gain de cause que le grand Guyanais eut dans son affaire avec le sinistre négrier Mury ${ }^{11} »$. Le 14 novembre 1936,

Maran répond à ma lettre aérienne du 27 écoulé. J'aime beaucoup la franchise de ce grand chasseur d'images et prestigieux paysagiste. C'est, d'ailleurs, pourquoi j'ai tenu, malgré la brutalité, et pour cause, de sa pensée, à le garder parmi ceux que j'appelle vraiment mes amis.

Maran s'est mépris sur mes véritables intentions contenues dans le billet auquel il répond. Et cela m'a valu une de ces lettres bourrues comme il en a le secret. Donc, il se refuse à prendre contact, pour moi, avec [Henri] Mariol. « Les milieux littéraires de Paris, m'affirme-t-il à peu près, me font vomir. Je vais faire quelque chose pour vous, mais en cavalier seul. » Et il me promet un article dans la Dépêche de Toulouse. C'est bien - et c'est, au reste, tout ce que j'ai voulu - avec, il est vrai, le désir de ne point voir se disperser les efforts tentés en ma faveur et plutôt de les voir se coordonner. Alors j'ai nommé Mariol.

Écrirai cela, mardi, au grand écrivain noir ${ }^{12}$.

Leur amitié est publique et se lit également dans la presse - medium dont ils font tous deux grand usage. Maran signe dans La Dépêche de Toulouse du 28 août 1934 un long portrait de Rabearivelo :

Le curieux homme que ce doit être, ce Jean-Joseph Rabearivelo. Ce hova n'a jamais quitté la Grande île. Mais on le sent passionné de tout ce qui est français et l'on se rend compte, à le lire, qu'il est pénétré de culture française au point qu'il a peu ou prou suivi l'influence des écoles poétiques qui essaient depuis quarante ans de tordre le cou à l'éloquence du romantisme [...]. Et ce sont des modulations douces, d'incertaines berceuses souvent presque naïves, de temps à autre précieuses jusqu'au gongorisme, des effusions d'âme comme susurrées et si vagues qu'il leur arrive de s'étonner elles-mêmes devant les brusques hiatus que créent leurs rêveries; enfin, des thèmes ou plutôt des élégies qui ne sont que beauté et pureté [...].

Si nos services de propagande étaient ce qu'on voudrait qu'ils fussent; si les plus importantes de nos sociétés littéraires s'intéressaient aux lettres françaises autant 
qu'elles prétendent le faire, il y a longtemps qu'on aurait donné à Jean-Joseph Rabearivelo, poète malgache de langue française, pour l'excellent travail qu'il poursuit dans son pays, la récompense qu'il n'a pas encore reçue, mais qu'il mérite. se prolonge cependant, comme le signalent les archives familiales ${ }^{15}$. Il possède dans sa bibliothèque des ouvrages comme La Grande île de Madagascar de Marius-Ary Leblond (Paris, Charles Delagrave, 1907 ${ }^{16}$ ). Il écrit des notes de lecture sur Madagascar depuis l'Occupation française: journal d'un administrateur ${ }^{17}$ de Georges Talvas, mais aussi sur Contes et Légendes de Madagascar de Renée Vally-Samat ou encore sur Le Livre de la Sagesse malgache de Elian J. Finbert. Et en 1948, en réponse à la répression très brutale du soulèvement malgache pour l'indépendance, il publiera un article sur « Le racisme et le colonialisme à l'œuvre, les événements de Madagascar $»^{18}$.

\section{NOTES}

1. Le Monde illustré, 21 août 1937.

2. « Un petit-neveu de Chatterton. Jean-Joseph Rabearivelo », Le Monde illustré, 21 août 1937.

3. Ce journal soutenait alors la mission d'enquête confiée par la Ligue française pour l'accession des indigènes de Madagascar aux droits des citoyens français au militant nationaliste Jean Ralaimongo.

4. «Enquête sur les prix Goncourt ", feuillet tapuscrit, TP.GONC, https://eman-archives.org/ francophone/items/show/2086; dans Jean-Joseph Rabearivelo, Euvres complètes, tome II : Le 
poète, le narrateur, le dramaturge, le critique, le passeur de langues, l'historien, édition critique coordonnée par Serge Meitinger, Laurence Ink, Liliane Ramarosoa et Claire Riffard, Paris, CNRS Éditions, coll. « Planète libre », $\mathrm{n}^{\circ}$ 3, 1789 p., 2012 ; (OC2), p. 1381-1382.

5. "Organe d'action du Théâtre d'Art libre », dirigée notamment par le maurassien Gérard de Catalogne.

6. «Enquête sur les prix Goncourt ", dans OC2, p. 1381-1282.

7. Serge Meitinger, «L'Aube rouge ou du sang sur le soleil », dans OC2, p. 805.

8. Également dédicacé à son grand-père le pasteur Andriambelo, ici orthographié Randriambelo, à Samuel Jafetra, écrivain et photographe, ami de Rabearivelo et oncle de sa future épouse Mary, et à l'écrivain Pierre Camo, premier mentor.

9. René Maran, «Gandhi », traduction d'Edna Worthley Underwood, Opportunity, $\mathrm{n}^{\circ} 3$, février 1925, p. 40-42.

10. L'expression est de Serge Meitinger, "Introduction", dans Euvres complètes, tome I : Le diariste (Les Calepins bleus), L'épistolier, Le moraliste, édition critique coordonnée par Serge Meitinger, Liliane Ramarosoa et Claire Riffard, Paris, CNRS Éditions / Présence Africaine, coll. «Planète libre », $\mathrm{n}^{\circ}$ 2, 1278 p., 2010 (OC1).

11. DansOC1, p. 865.

12. DansOC1, p. 1057-1058.

13. La Dépêche de Toulouse, 8 décembre 1936.

14. René Maran, «Gandhi », traduction d'Edna Worthley Underwood, Opportunity, février 1925, p. 40-42, et "The Harriet Beecher Stowe of France ", traduction d'Edna Worthley Underwood, Opportunity, août 1925, p. 229-231. En 1926, Charles S. Johnson et Alain Locke soumettent le Roman d'un Negre (traduit à cet effet par Mrs. Underwood) au Albert \& Charles Boni Novel Competition. Voir la lettre de Locke à Maran du 23 déc. 1926, Alain Locke Papers, Moorland-Spingarn Research Center, Howard University.

15. Tous mes remerciements à Bernard Michel pour les informations communiquées à ce sujet.

16. Je remercie Mbaye Gueye pour cette information.

17. Grandes éditions de Paris, 1939.

18. Jeune République $\mathrm{n}^{\circ}$ 6-1064, 01/09/1948.

\section{RÉSUMÉS}

Le 22 juin 1937, juste avant de se donner la mort dans son île australe, à 34 ans seulement, l'écrivain malgache Jean-Joseph Rabearivelo écrivait une dernière lettre à son ami René Maran, qui répondait, avec émotion, dans un hommage posthume. Ce dialogue amical poursuivi durant plus de dix ans propose quelques éléments inédits sur les positions de Maran en matière politique et littéraire.

On June 22, 1937, just before he chose to die on his Southern Island, at only 34 years of age, the Malagasy writer Jean-Joseph Rabearivelo wrote a last letter to his friend René Maran, who responded with emotion in a posthumous tribute. This friendly dialogue, which lasted for more than ten years, offers some new elements on Maran's political and literary positions. 
INDEX

Mots-clés : René Maran, Jean-Joseph Rabearivelo, Chatterton, Edna Worthley Underwood

Keywords : René Maran, Jean-Joseph Rabearivelo, Chatterton, Edna Worthley Underwood

\section{AUTEUR}

\section{CLAIRE RIFFARD}

Responsable de l'équipe « manuscrits francophones » de l'ITEM (CNRS-ENS) 